\title{
PERRON-FROBENIUS THEOREM FOR MULTI-VALUED MAPPINGS
}

\author{
HIROSHI TATEISHI
}

\section{Introduction}

The Perron-Frobenius theorem concerning eigenvalues of nonnegative matrices has been extended to several directions; e.g. (i) extension to nonlinear mappings (Fujimoto [5], Fujimoto and Morishima [7], Morishima [13], Nikaido [15], Oshime [16], [17], Samuelson and Solow [20]), (ii) extension to positive linear operators defined on infinite dimensional spaces (Karlin [8], Niiro and Sawashima [14], Shaefer [21]), and (iii) extension to multi-valued mappings (Aubin [1], [2], Aubin and Ekeland [3], Aubin and Frankowska [4], Fujimoto and Herrero [7], Makarov and Rubinov [11], Rockafellar [18], [19]).

In this paper, we shall primarily be concerned with the third category. The multi-valued versions of the Perron-Frobenius theorem have been motivated chiefly by von Neumann's theory of dynamic economic growth (von Neumann [25]). Let $G: \boldsymbol{R}_{+}^{n} \rightarrow \rightarrow \boldsymbol{R}_{+}^{n}$ be a multi-valued mapping with a conic graph. This mapping is interpreted as a relation which associates with each input vector the set of technologically possible output vectors of the whole economy. A sequence $\left(x_{t}\right)_{t=0}^{\infty} \subseteq \prod_{t=0}^{\infty} \boldsymbol{R}_{+}^{n}$ which satisfies the condition :

$$
x_{t+1} \in G\left(x_{t}\right) \quad \text { for all } t=0,1,2, \cdots
$$

is called a feasible path of this economy. When a feasible path $\left(x_{t}\right)$ is represented, in particular, by $x_{t}=\lambda^{t} x_{0}$ for some $x_{0} \in \boldsymbol{R}_{+}^{n}$ and $\lambda \geqq 0$, it is called a balanced growth path with growth factor $\lambda$. A balanced growth path which attains the maximum growth factor enjoys several desired properties from a viewpoint of normative economic theory. It should be noted that the study of the balanced growth path is, thanks to the conicity of the graph of $G$, reduced to the study of $x_{0} \in \Sigma^{n}$ and $\lambda \geqq 0$ which satisfy the condition:

$$
\lambda x_{0} \in G\left(x_{0}\right)
$$

(where $\Sigma^{n}$ is the fundamental simplex in $\boldsymbol{R}^{n}$ ). It is nothing other than the multi-valued version of eigenvalue problem. Here emerges a natural incentive for inquiring the multi-valued version of Perron-Frobenius theorem.

Received March 15, 1991. 
Our results are closely related with those of Aubin and Ekeland [3]. Although their fundamental theorem on the existence of maximum eigenvalue is formulated under very general conditions, its proof seems to contain some mistakes in subtle points (Aubin and Ekeland [3], p. 147, Proposition 1). In particular, their treatment of fractions with 0 denominator is quite dubious. The purpose of the present paper is to provide a modified correct version of their result with a new proof, which is totally different from theirs and is essentially based upon the minimax reasoning.

\section{Notations and Assumptions}

Let $\boldsymbol{R}^{n}$ be the $n$ dimensional Euclidean space and $\boldsymbol{R}_{+}^{n}$ its nonnegative orthant. We denote by $\Sigma^{n}$ the fundamental simplex of $\boldsymbol{R}^{n}$. For any $A \subset \boldsymbol{R}^{m}$ and $p \in \boldsymbol{R}^{m}$, we denote by $\boldsymbol{\sigma}(A, p)$ the support functional of $A$; i.e. $\boldsymbol{\sigma}(A, p)=$ $\sup \{\langle x, p\rangle: x \in A\}$, where $\langle\cdot, \cdot\rangle$ designates the usual inner product.

A single-valued mapping $f: \Sigma^{n} \longrightarrow \boldsymbol{R}_{+}^{m}$ as well as multi-valued mapping $G: \Sigma^{n} \rightarrow \rightarrow \boldsymbol{R}_{+}^{m}$ are assumed to be given. The conditions imposed on these mappings are as follows. We denote by $f_{\imath}$ the $i$-th coordinate of $f$.

A1. $f_{\imath}: \Sigma^{n} \longrightarrow \boldsymbol{R}_{+}$is quasi-convex and lower semi-continuous for all $i=$ $1,2, \cdots, n$.

A2. (i) The set $G(x)$ is non-empty, compact and convex for all $x \in \Sigma^{n}$.

(ii) The function $x \longmapsto \sigma(G(x), p)$ is quasi-concave and upper semicontinuous for any fixed $p \in \Sigma^{m}$.

A3. For any $p \in \Sigma^{m}$, there exists $x \in \Sigma^{n}$ such that $\sigma(G(x), p)>0$.

A4. There exists $p \in \Sigma^{m}$ such that $\langle p, f(x)\rangle>0$ for all $x \in \Sigma^{n}$.

Remark. These assumptions are approximately corresponding to those considered in Aubin and Ekeland [3] (p. 147, Proposition 1). They admit $f_{\imath}$ to take negative values, but assume that it is a convex function. And instead of our assumptions of the quasi-concavity and the upper semi-continuity of $\sigma(G(\cdot), p)$, they assume the convexity of the graph of $G$ and upper semicontinuity of $G$ in the sense of multi-valued mappings. (For the analysis of multi-valued mappings, see Aubin-Frankowska [4] or Maruyama [12].) It is easy to verify that, under the condition that $G$ is the compact-valued mapping, their assumptions imply ours except for the nonnegativity. A merit of our modification of the assumptions concerning $G$ is that, by getting rid of the assumption that the graph of $G$ is convex, our results can be directly applied to the cases where $G$ is a single-valued nonlinear mapping as shown in section 4.

\section{Main Theorems}

Theorem 1. Let $f$ and $G$ satisfy assumptions $A 1, A 2, A 3$ and $A 4$. Then there exist $\delta>0, p^{*} \in \Sigma^{m}$ and $x^{*} \in \Sigma^{n}$ which satisfy the following four conditions: 
(i ) $\delta f\left(x^{*}\right) \in G\left(x^{*}\right)-\boldsymbol{R}_{+}^{m}$.

(ii) $\sigma\left(G(x), p^{*}\right) \leqq \delta\left\langle p^{*}, f(x)\right\rangle$ for all $x \in \Sigma^{n}$.

(iii) $\sigma\left(G\left(x^{*}\right), p^{*}\right)=\delta\left\langle p^{*}, f\left(x^{*}\right)\right\rangle$.

(iv) For any $\lambda>0$ which satisfies the condition: $\lambda f(x) \in G(x)-\boldsymbol{R}_{+}^{m}$ for some $x \in \Sigma^{n}$, we have $\lambda \leqq \delta$.

Proof. We begin by distinguishing two kinds of definitions of fractions with 0 denominators. $a / b$ is defined as

$$
\frac{a}{b}= \begin{cases}a / b \text { (in the usual sense) } & \text { if } b \neq 0 \\ +\infty & \text { if } b=0 .\end{cases}
$$

On the other hand, $a{ }^{*} b$ is defined as

$$
\frac{a}{b} *= \begin{cases}a / b(\text { in the usual sense) } & \text { if } b \neq 0 \\ 0 & \text { if } a=b=0 \\ +\infty & \text { if } a \neq 0, b=0 .\end{cases}
$$

Step 1. Define a number $\delta$ by

$$
\delta=\inf _{p \in \Sigma^{m}} \sup _{x \in \Sigma^{n}} \frac{\sigma(G(x), p)}{\langle p, f(x)\rangle} .
$$

Then $\delta$ is a well-defined positive real number. In order to see this, let $S: \Sigma^{m} \longrightarrow$ $(0,+\infty]$ be a function defined by

$$
S(p)=\sup _{x} \frac{\sigma(G(x), p)}{\langle p, f(x)\rangle} .
$$

Furthermore let $\hat{p} \in \Sigma^{m}$ be fixed so that it satisfies $A 4$. Then the function $T: \Sigma^{n} \rightarrow \boldsymbol{R}_{+}$defined by

$$
T(x)=-\frac{\sigma(G(x), \hat{p})}{\langle\hat{p}, f(x)\rangle}
$$

is upper semi-continuous and thus has a maximum point. So $S$ is proper, i. e., it is not identically $+\infty$, and $\delta$ is a nonnegative real number. Finally we show that $\delta$ is in fact positive. To see this, we define $U(p)$ for any $p \in \Sigma^{m}$ by

$$
U(p)=\sup _{x} \frac{\sigma(G(x), p)}{\langle p, f(x)\rangle} * .
$$

$U(p)$ belongs to $(0,+\infty]$ by $A 3$. Since it is clear that

$$
U(p) \leqq S(p) \quad \text { for all } p \in \Sigma^{m},
$$

$U$ is also proper. The lower semi-continuity of $U$ follows from the fact that the set 


$$
\begin{aligned}
& \left\{p \in \Sigma^{m}: U(p) \leqq \alpha\right\} \\
& =\left\{p \in \Sigma^{m}: \sigma(G(x), p) \leqq \alpha\langle p, f(x)\rangle \quad \text { for all } x \in \Sigma^{n}\right\} \\
& =\cap\left\{p \in \Sigma^{m}: \sigma(G(x), p) \leqq \alpha\langle p, f(x)\rangle\right\}
\end{aligned}
$$

(where $\cap$ is taken over all $x \in \Sigma^{n}$ ) is closed for any $\alpha>0$. Thus $U$ is a proper and lower semi-continuous function defined on the compact set $\Sigma^{m}$. So it has the positive minimum, and hence $\delta$ is positive.

Step 2. Using $\delta$ defined in Step 1, we define a function $\phi: \Sigma^{m} \times \Sigma^{n} \longrightarrow \boldsymbol{R}$ as follows,

$$
\phi(p, x)=\sigma(G(x), p)-\delta\langle p, f(x)\rangle .
$$

Then it can easily be verified that for each fixed $x \in \Sigma^{n}$, the function $p \longmapsto \phi(p, x)$ is lower semi-continuous and quasi-convex and that for each fixed $p \in \Sigma^{m}$, the function $x \longmapsto \phi(p, x)$ is upper semi-continuous and quasi-concave. Therefore by Sion's minimax theorem (c.f. Sion [22] or 'Takahashi [23]), $\phi$ has a minimax point $\left(p^{*}, x^{*}\right) \in \Sigma^{m} \times \Sigma^{n}$.

We now proceed to show that $\phi\left(p^{*}, x^{*}\right)=0$. To this end, we show that both $\phi\left(p^{*}, x^{*}\right) \geqq 0$ and $\phi\left(p^{*}, x^{*}\right) \leqq 0$ are valid.

(a) The proof of $\phi\left(p^{*}, x^{*}\right) \geqq 0$.

Case 1. $\left\langle p^{*}, f(x)\right\rangle>0$ for all $x \in \Sigma^{n}$.

Let $x^{\prime}$ be a maximum point of the function

$$
x \longmapsto \frac{\sigma\left(G(x), p^{*}\right)}{\left\langle p^{*}, f(x)\right\rangle} .
$$

Then, it follows that

$$
\begin{aligned}
\phi\left(p^{*}, x^{*}\right) \\
\quad=\min _{p} \max _{x}[\sigma(G(x), p)-\delta\langle p, f(x)\rangle] \\
\quad=\max _{x}\left[\sigma\left(G(x), p^{*}\right)-\left\{\inf _{p} \sup _{x} \frac{\sigma(G(x), p)}{\langle p, f(x)\rangle}\right\}\left\langle p^{*}, f(x)\right\rangle\right] \\
\quad \geqq \max _{x}\left[\sigma\left(G(x), p^{*}\right)-\left\{\sup _{x} \frac{\sigma\left(G(x), p^{*}\right)}{\left\langle p^{*}, f(x)\right\rangle}\right\}\left\langle p^{*}, f(x)\right\rangle\right] \\
\quad=\max _{x}\left[\sigma\left(G(x), p^{*}\right)-\frac{\sigma\left(G\left(x^{\prime}\right), p^{*}\right)}{\left\langle p^{*}, f\left(x^{\prime}\right)\right\rangle}\left\langle p^{*}, f(x)\right\rangle\right] \\
\geqq \sigma\left(G\left(x^{\prime}\right), p^{*}\right)-\frac{\sigma\left(G\left(x^{\prime}\right), p^{*}\right)}{\left\langle p^{*}, f\left(x^{\prime}\right)\right\rangle}\left\langle p^{*}, f\left(x^{\prime}\right)\right\rangle \\
=0 .
\end{aligned}
$$

Case 2. $\left\langle p^{*}, f\left(x^{\prime \prime}\right)\right\rangle=0$ for some $x^{\prime \prime} \in \Sigma^{n}$.

In this case, we also obtain the relation: 


$$
\begin{aligned}
\phi\left(p^{*}, x^{*}\right) & =\min _{p} \max _{x}[\sigma(G(x), p)-\delta\langle p, f(x)\rangle\} \\
& =\max _{x}\left[\sigma\left(G(x), p^{*}\right)-\delta\left\langle p^{*}, f(x)\right\rangle\right] \\
& \geqq \sigma\left(G\left(x^{\prime \prime}\right), p^{*}\right)-\delta\left\langle p^{*}, f\left(x^{\prime \prime}\right)\right\rangle \\
& =\sigma\left(G\left(x^{\prime \prime}\right), p^{*}\right) \\
& \geqq 0 .
\end{aligned}
$$

(b) The proof of $\phi\left(p^{*}, x^{*}\right) \leqq 0$. Let $p^{\prime} \in \Sigma^{m}$ be the minimum point of $U$ obtained in Step 1.

Case 1. $\left\langle p^{\prime}, f\left(x^{*}\right)\right\rangle>0$.

We can get the desired inequality by the simple calculation:

$$
\begin{aligned}
\phi\left(p^{*},\right. & \left.x^{*}\right) \\
& =\max _{x} \min _{p}[\sigma(G(x), p)-\delta\langle p, f(x)\rangle] \\
& =\min _{p}\left[\sigma\left(G\left(x^{*}\right), p\right)-\delta\left\langle p, f\left(x^{*}\right)\right\rangle\right] \\
& \leqq \min _{p}\left[\sigma\left(G\left(x^{*}\right), p\right)-\left\{\inf _{p} \sup _{x} \frac{\sigma(G(x), p)}{\langle p, f(x)\rangle} *\right\}\left\langle p, f\left(x^{*}\right)\right\rangle\right] \\
& =\min _{p}\left[\sigma\left(G\left(x^{*}\right), p\right)-\left\{\sup _{x} \frac{\sigma\left(G(x), p^{\prime}\right)}{\left\langle p^{\prime}, f(x)\right\rangle} *\right\}\left\langle p, f\left(x^{*}\right)\right\rangle\right] \\
& \left.\leqq \sigma\left(G\left(x^{*}\right), p^{\prime}\right)-\left\{\sup _{x} \frac{\sigma\left(G(x), p^{\prime}\right)}{\left\langle p^{\prime}, f(x)\right\rangle} *\right\} p^{\prime}, f\left(x^{*}\right)\right\rangle \\
& \leqq \sigma\left(G\left(x^{*}\right), p^{\prime}\right)-\frac{\sigma\left(G\left(x^{*}\right), p^{\prime}\right)}{\left\langle p^{\prime}, f\left(x^{*}\right)\right\rangle}\left\langle p^{\prime}, f\left(x^{*}\right)\right\rangle \\
& =0 .
\end{aligned}
$$

Case 2. $\left\langle p^{\prime}, f\left(x^{*}\right)\right\rangle=0$.

In this case, $\sigma\left(G\left(x^{*}\right), p^{\prime}\right)=0$ by the definition of $U$. Hence we must have:

$$
\begin{aligned}
\phi\left(p^{*}, x^{*}\right) & =\max _{x} \min _{p}[\sigma(G(x), p)-\delta\langle p, f(x)\rangle] \\
& =\min _{p}\left[\sigma\left(G\left(x^{*}\right), p\right)-\delta\left\langle p, f\left(x^{*}\right)\right\rangle\right] \\
& \leqq \sigma\left(G\left(x^{*}\right), p^{\prime}\right)-\delta\left\langle p^{\prime}, f\left(x^{*}\right)\right\rangle \\
& =0 .
\end{aligned}
$$

This completes the proof of the equality $\phi\left(p^{*}, x^{*}\right)=0$.

Step 3. We show that the minimax point $\left(p^{*}, x^{*}\right)$ of $\phi$ together with $\delta>0$ in fact satisfies all the required conditions. $\phi\left(p^{*}, x^{*}\right)=0$ means that $\left(p^{*}, x^{*}\right)$ 
satisfies the conditions (ii), (iii) and the following inequality.

$$
\sigma\left(G\left(x^{*}\right), p\right)-\delta\left\langle p, f\left(x^{*}\right)\right\rangle \geqq 0 \quad \text { for all } p \in \Sigma^{m} .
$$

This condition, in turn, implies condition ( $i)$. To see this, suppose, on the contrary, that $\delta$ and $x^{*}$ do not satisfy condition (i), that is,

$$
\delta f\left(x^{*}\right) \notin G\left(x^{*}\right)-\boldsymbol{R}_{+}^{m} .
$$

Then by the separation theorem, we must have

$$
\sigma\left(G\left(x^{*}\right)-\boldsymbol{R}_{+}^{m}, q\right)-\delta\langle q, f(x)\rangle<0 \quad \text { for some } q \in \boldsymbol{R}^{m}, q \neq 0 .
$$

This $q$ can be taken from $\Sigma^{m}$ thanks to the term "- $\boldsymbol{R}_{+}^{m}$ ". Thus follows the inequality.

$$
\sigma\left(G\left(x^{*}\right), q\right)-\delta\left\langle q, f\left(x^{*}\right)\right\rangle<0 .
$$

But this contradicts to (1).

Finally, we check the condition (iv). To this end, choose $\lambda>0$ so that

$$
\lambda f\left(x^{\prime}\right) \in G\left(x^{\prime}\right)-\boldsymbol{R}_{+}^{m} \quad \text { for some } \quad x^{\prime} \in \Sigma^{n} .
$$

Then it follows that

$$
\sigma\left(G\left(x^{\prime}\right), p\right)-\lambda\left\langle p, f\left(x^{\prime}\right)\right\rangle \geqq 0 \quad \text { for all } p \in \Sigma^{m} .
$$

Therefore, we must have the relation

$$
\lambda \leqq \frac{\sigma\left(G\left(x^{\prime}\right), p\right)}{\left\langle p, f\left(x^{\prime}\right)\right\rangle} \leqq \sup _{x} \frac{\sigma(G(x), p)}{\langle p, f(x)\rangle} \quad \text { for all } p \in \Sigma^{m} .
$$

Hence we can conclude that

$$
\lambda \leqq \inf _{p} \sup _{x} \frac{\sigma(G(x), p)}{\langle p, f(x)\rangle}=\delta .
$$

This completes the proof of the theorem.

\section{Some Consequences}

In this section, we discuss some important consequences of our main theorem. Although they are essentially the same as those obtained by AubinEkeland [3], we collect them here again for the sake of readers' conveniences.

First of all, we give the conditions which are necessary to guarantee the existence of $x \in \Sigma^{n}$ satisfying the following condition:

$$
\delta f(x) \in G(x) .
$$

For this purpose, we need to strengthen some assumptions imposed on $f$ and $G$ as follows. 
$A 3^{\prime}$. For any $p \in \Sigma^{m}$ and $x \in \Sigma^{n}$, there exists $z \in G(x)$ such that $\langle p, z\rangle>0$, $A 4^{\prime}$. There exists $p \in$ int. $\boldsymbol{R}_{+}^{m}$ such that $\langle p, f(x)\rangle>0$ for all $x \in \Sigma^{n}$.

A5. $m=n$ and for any $i=1,2, \cdots, n, x_{\imath}=0$ implies $f_{i}(x) \leqq 0$.

Proposition 1. Let $f$ and $G$ satısfy assumptions $A 1, A 2, A 3^{\prime}, A 4^{\prime}$ and $A 5$. Then there exist $\delta>0, p^{*} \in$ int. $\Sigma^{n}$ and $x^{*} \in \Sigma^{n}$ which satisfy (ii), (iii), (iv) and the inclusion:

$$
\delta f\left(x^{*}\right) \in G\left(x^{*}\right)
$$

The next proposition should be regarded as a generalized version of the nonnegative invertibility of the nonnegative matrices.

Proposition 2. Let $f$ and $G$ satısfy assumptions $A 1, A 2, A 3$ and $A 4$. We We define a number $\beta<0$, for any $\mu>\delta$ and $y \in$ int. $\boldsymbol{R}_{+}^{m}$, by

$$
\beta=\inf _{p} \sup _{x} \frac{\sigma(G(x)-\mu f(x), p)}{\langle p, y\rangle} .
$$

Then there exists $x^{*} \in \Sigma^{n}$ such that

$$
\beta y \in G\left(x^{*}\right)-\mu f\left(x^{*}\right)-\boldsymbol{R}_{+}^{m} .
$$

If we assume $A 1, A 2, A 3^{\prime}, A 4^{\prime}$ and $A 5$, then the term "-- $\boldsymbol{R}_{+}^{m}$ " can be dropped.

Proposition 3. Let $f$ and $G$ satisfy assumptions $A 1, A 2, A 3^{\prime}, A 4^{\prime}$ and $A 5$. We define $\beta<0$, for any $\mu>\delta$ and $y \in$ int. $\boldsymbol{R}_{+}^{n}$, by

$$
\beta=\inf _{p} \sup _{x} \frac{\sigma(G(x)-\mu f(x), p)}{\langle p, y\rangle} .
$$

Then there exists $x^{*} \in \Sigma^{n}$ such that

$$
\beta y \in G\left(x^{*}\right)-\mu f\left(x^{*}\right) .
$$

Finally, we give consequences in the case $G$ is single-valued.

COROLlARY 1. Let $f$ and $g: \Sigma^{n} \longrightarrow \boldsymbol{R}_{+}^{m}$ be single-valued mappings which satisfy the following conditions.

(1) $f_{\imath} \imath$ s quasi-convex and lower semı-contınuous for all $\imath=1,2, \cdots, m$.

(2) $g_{\imath}$ is quasi-concave and upper semi-continuous for all $\imath=1,2, \cdots, m$.

(3) For any $p \in \Sigma^{m}$, there exists $x \in \Sigma^{n}$ such that $\langle p, g(x)\rangle>0$.

(4) There exists $p \in \Sigma^{m}$ such that $\langle p, f(x)\rangle>0$ for all $x \in \Sigma^{n}$.

Then there exist $\delta>0, p^{*} \in \Sigma^{m}$ and $x^{*} \in \Sigma^{n}$ such that

(i) $\delta f\left(x^{*}\right) \leqq g\left(x^{*}\right)$.

(ii) $\left\langle p^{*}, g(x)\right\rangle \leqq \delta\left\langle p^{*}, f(x)\right\rangle$ for all $x \in \Sigma^{n}$.

(iii) $\left\langle p^{*}, g\left(x^{*}\right)\right\rangle=\delta\left\langle p^{*}, f\left(x^{*}\right)\right\rangle$.

(iv) For any $\lambda>0$ which satisfies the condition $\lambda f(x) \leqq g(x)$ for some $x E$ $\Sigma^{n}$ we have $\lambda \leqq \delta$. 
(v) For any $\mu>\delta$ and $y \in$ int. $\boldsymbol{R}_{+}^{m}$, there exist $\beta<0$ and $x^{\prime} \in \Sigma^{n}$ such that $\beta y \leqq g\left(x^{\prime}\right)-\mu f\left(x^{\prime}\right)$.

The next corollary is an extended version of the Perron-Frobenius theorem to nonlinear mapping.

COROLLARY 2. Let $g: \Sigma^{n} \longrightarrow \boldsymbol{R}_{+}^{n}$ be a single-valued mapping which satisfy the following conditons.

(1) $g_{\imath}$ is quasi-concave and upper semı-continuous for all $\imath=1,2, \cdots, n$.

(2) $g(x) \gg 0$ for all $x \in \Sigma^{n}$.

Then there exist $\delta>0$ and $p^{*}, x^{*} \in$ int. $\Sigma^{n}$ such that

(i ) $\delta x^{*}=g\left(x^{*}\right)$.

(ii) $\left\langle p^{*}, g(x)\right\rangle \leqq \delta\left\langle p^{*}, x\right\rangle$ for all $x \in \Sigma^{n}$.

(iii) $\left\langle p^{*}, g\left(x^{*}\right)\right\rangle=\delta\left\langle p^{*}, x^{*}\right\rangle$

(iv) For any $\lambda>0$ which satisfies the condition: $\lambda x \leqq g(x)$ for some $x \in \Sigma^{n}$ we have $\lambda \leqq \delta$.

(v) For any $\mu>\delta$ and $y \in$ int. $\boldsymbol{R}^{n}$, there exzst $\beta<0$ and $x^{\prime} \in$ int. $\Sigma^{n}$ such that $\beta y=g\left(x^{\prime}\right)-\mu x^{\prime}$.

Remark We mention here a few remarks about the relationships between corollary 2 and the other results of the nonlinear versions of the PerronFrobenius theorem. The typical assumptions imposed on $g: \boldsymbol{R}_{+}^{n} \longrightarrow \boldsymbol{R}_{+}^{n}$ are (1) continuity, (2) homogeneity, (3) monotonicity, and (4) indecomposability. Under these assumptions, it can be shown that there exists maximum eigenvalue and that this eigenvalue satisfies the property (iv) in corollary 2. To put it minutely, the existence of the eigenvalues can be proved under assumption (1), and the existence of the maximum eigenvalue can be proved under assumptions (1) and (2). Furthermore if we assume (3) in addition to (1) and (2), it can be shown that the maximum eigenvalue satisfies the condition (iv) in corollary 2, and lastly if we assume (3) and (4) in addition to (1) and (2), the maximum eigenvalue can be shown to be positive. (See, for example, Nikaido [14].) Under our results, assumption (1) is weakened to the upper semi-continuity of $g$ and assumption (4) is strengthened to the strict positivity of $g$. On the other hand, assumptions (2) and (3) are incomparable with ours, because our results restrict the domain of $g$ to $\Sigma^{n}$ from the outset.

Finally if $G$ is a matrix, our result is reduced to the well-known Perron theorem.

COROllary 3. Let $g: \boldsymbol{R}^{n} \longrightarrow \boldsymbol{R}^{n}$ be a positive matrix. Then the following conditions hold.

(i) $g$ has a positive eigenvalue $\delta$ with the corresponding ergenvector $x^{*}$ with posituve components.

(ii) $\delta$ is the only eigenvalue of $g$ for which there corresponds an eigenvector $x \in \Sigma^{n}$. 

of $g$.

(iii) $\delta$ is larger than or equal to the absolute value of any other ergenvalue

(iv) The matrix $\mu I-g$ is invertible and $(\mu I-g)^{-1}$ is positive if and only if $\mu>\delta$, where $I$ is the identity matrix.

\section{REFERENCE}

[1] Aubin, J.-P., Propriété de Perron-Frobenius pour des correspondances, C.R. Acad. Sc. Paris. 286 (1978), 911-914.

[2] Aubin, J.-P., Applied functional analysis, Wiley, N. Y., 1979.

[3] Aubin, J.-P. and I. Ekeland, Applied nonlinear analysis, Wiley, N.Y., 1984.

[4] Aubin, J.-P. and H. Frankowska, Set-valued analysis, Birkhäuser, Boston, 1990.

[5] Fujimoto, T., Nonlinear generalization of the Frobenius theorem, J. Math. Eco. 6 (1979), 17-21.

[6] Fujimoto, T. and C. Herrero, The Perron-Frobenius theorem for set-valued mappings, Kagawa daıgaku keızai ronso 59 (1988), 234-245.

[7] Fujimoto, T. and M. Morishima, The Frobenius theorem, its Solow-Samuelson extension and the Kuhn-Tucker theorem, J. Math. Eco. 1 (1974), 199-205.

[8] Karlin, S., Positive operators, Math. Mech. 8 (1959), 907-937.

[9] Kemeny, J., O. Morgenstern and G. Thompson, A generalization of von Neumann model of an expanding economy, Econometrica 24 (1956), 115-135.

[10] KrasnoselskiI, M.A., Positive solutions of operator equations, Noordhoff, Groningen, 1964.

[11] Makarov, V.L. and A.M. Rubinov, Mathematical theory of economic dynamics and equilibria, Springer, N. Y., 1977.

[12] Maruyama, T., Nonlinear analysis in economic equilibrium theory (Kinkou bunseki no suuri), Nihonkeizai Shinbunsha, Tokyo, 1985 (in Japanese).

[13] Morishima, M., Equilibrium stability and growth, Clarendon Press, Oxford, 1964.

[14] Nirro, F. and I. Sawashima, On the spectral properties of positive irreducible operators in An arbitrary Banach lattice and problem of H.H. Schaefer, Sci. Pap. of College Gen. Educ. Univ. Tokyo 16 (1966), 145-183.

[15] Nikaido, H., Convex structures and economic theory, Academic Press, N.Y., 1968.

[16] Oshime, Y., An extension of Morishima's nonlinear Perron-Frobenius theorem, J. Math. Kyoto Univ., 23-4 (1983), 803-830.

[17] Oshime, Y., Non-linear Perron-Frobenius problem for weakly contractive transformations, Math. Japonica, 29 (1984), 681-704.

[18] Rockafellar, R., Monotone process of convex and concave type, Memorrs of the American Mathematical Society, 77 (1967).

[19] RockafellaR, R., Convex algebra and duality in dynamic models of production, in Mathematical models in economics, ed. by J. Los and M. W. Los, American Elsevier Publishing Company, N. Y., 1974.

[20] Samuelson, P.A. and R. M. Solow, Balanced growth under constant returns to scale, Econometrica, 21 (1953), 412-424.

[21] Schaefer, H.H., Banach lattices and positive operators, Springer, Berlin, 1974.

[22] Sion, M., On general minimax theorem, Pacific J. Math., 8 (1958), 171-176.

[23] TAKAHASHI, W., Nonlinear variational inequalities and fixed point theorems. J. 
Math. Soc. Japan, 28 (1976), 168-181.

[24] TAKAhashi, W., Nonlinear functional analysis (Hisenkei kansu kaisekigaku), Kindai Kagakusha, Tokyo, 1988 (in Japanese).

[25] von Neumann, J., Über ein Ökonomisches Gleichungssystem und eine Verallgemeinerung des Brouwerschen Fixpunktsatzes, Ergebnisse eines Mathematischen Kolloquiums, 8 (1937).

c/o Prof. Toru Maruyama

DEPARTMENT OF ECONOMICS

KEIO UNIVERSITY

2-15-45, Mita, Minato-KU

TOKYO 108, JAPAN 